\title{
The cerebral circulation
}

\author{
JAMES E. ECKENHOFF \\ M.D., D.Sc., F.F.A.R.C.S.
}

Northwestern University Medical School, Chicago, Illinois

THERE are three principal determinants of blood flow across the brain; the volume and pressure of the arterial supply, the resistance to the flow of blood across the brain, and the resistance to venous outflow.

Two paired arteries, the carotids and vertebrals, deliver about $750 \mathrm{ml}$ of blood/min to the brain, or about $12 \%$ of the cardiac output. In hypovolaemic states a relatively larger portion of the cardiac output goes to the brain. The four arteries deliver blood into the circle of Willis, a vascular entity which allows, under some circumstances, blood to flow in a retrograde fashion into any one of the four arteries in which there is an obstruction proximal to the origin of the vessel. However, the entire circle of Willis is patent in only $10-15 \%$ of individuals.

Arterial blood supply. The perfusing pressure of blood at the base of the brain is variable depending upon body position. When supine with the head level with the heart, the mean perfusing pressure would be the same as that measured in the brachial artery with the arm lying at the side. However, perfusing pressure is markedly reduced when the body is erect since the forces of gravity come into play. For each centimetre the point of measurement is above heart level, the mean blood pressure is $1 \mathrm{mmHg}$ less than that at heart level; if the mean pressure at heart level is $85 \mathrm{mmHg}$ and there is $35 \mathrm{~cm}$ distance from the base of the heart to the base of the brain, then the mean perfusing pressure would be $50 \mathrm{mmHg}$.

Under normal circumstances man can arise from the supine to the erect position with surprisingly little alteration of cerebral blood flow. This is provided there is not a significant reduction in cardiac output. Compensation can fail in the presence of severe hypovolaemia or under conditions which lead to peripheral pooling of blood such as orthostatic hypotension.

Cerebral resistance to blood flow. The resistance to the passage of blood across the brain has several determinants. From what has already been said it is obvious that baroreceptors of some sort are involved since marked changes in perfusing pressure fail to cause significant change in cerebral blood flow. It is generally acknowledged that adrenergic nerves do not control the cerebral circulation, although the large vessels at the base of the brain are subject to adrenergic control which upon stimulation may account for some small change in cerebral flow, although not a significant one.

Carbon dioxide. The principle determinant of flow is the tension of carbon dioxide in blood, vascular constriction occurring as the $\mathrm{PCO}_{2}$ decreases, and dilation following elevations in $\mathrm{PCO}_{2}$. Between 20-60 $\mathrm{mmHg}$ there is a linear relationship between $\mathrm{PCO}_{2}$ and flow; a $1 \mathrm{~mm}$ change in tension leads to a $1 \mathrm{ml}$ change in flow. The effect appears similarly in the vessels of the spinal cord. Maximal vasodilation occurs when the $\mathrm{PCO}_{2}$ is somewhere between 80 and $120 \mathrm{mmHg}$ and maximum vasoconstriction occurs 0 between 10 and $20 \mathrm{mmHg}$. The latter is associated with signs of cerebral hypoxia. Carbon dioxide exerts this control by virtue of its rapid diffusibility into extracellular fluid, changing the $\mathrm{pH}$, thus affecting vascular tone. If the $\mathrm{PCO}_{2}$ is kept constant and blood $\mathrm{pH}$ changed acutely, the effect on vascular tone comes on slowly, presumably because of slower acid diffusion.

Oxygen tension. Changes in oxygen tension of arterial blood through the normal ranges do not affect cerebral blood flow so long as there is not a concomitant change in $\mathrm{PCO}_{2}$. Thus, between 50 and $700 \mathrm{mmHg} \mathrm{Po}_{2}$, cerebral flow is constant. Below $\mathrm{Po}_{2}$ of $50 \mathrm{mmHg}$, vasodilation occurs presumably owing to the accumulation of acid metabolites. Such a dilator effect can be overruled by a change in $\mathrm{PCO}_{2}$. Above one atmosphere of oxygen, there is a progressive decrease in cerebral flow and at $2 \mathrm{~atm}$, the flow is reduced by $20 \%$. Under these circumstances, cerebral venous blood may be fully saturated with oxygen.

Cerebral metabolism. Under normal conditions, cerebral metabolism has minimal effect on cerebral flow. On average $3.0 \mathrm{ml}$ of oxygen is required per $100 \mathrm{~g}$ brain/min, a requirement that does not vary much from the awake to the asleep state. Although the effects of anaesthetics on cerebral vascular tone vary, the degree of effect is slight where compared 
with that of changes in $\mathrm{PCO}_{2}$, or of hypoxia, or of body temperature. There is a linear relationship between cerebral blood flow, cerebral metabolic rate for oxygen, and body temperature. $\mathrm{A} 10^{\circ} \mathrm{C}$ fall in body temperature will reduce flow and metabolic rate by $50 \%$.

It should be remembered that the invasive methods of measuring cerebral blood flow provide data averaged for the entire brain over a period of minutes. Although $44 \mathrm{ml}$ flow $/ 100 \mathrm{~g}$ brain/min is the usually quoted figure, there are regional variations with white matter having a flow rate of about $20 \mathrm{ml} / 100 \mathrm{~g} / \mathrm{min}$ and grey matter yielding rates of about $80 \mathrm{ml} / 100 \mathrm{~g} /$ min. Some variation has been noted with changing age, highest flows being recorded in the young. There does not appear to be a change from middle age to old age, although marked decreases have been noted in those with strokes or senile dementia.

The striking difference in the coronary and cerebral circulations is worth keeping in mind. The heart is a working muscle, it has a high blood flow rate and extracts most of the oxygen from the blood delivered to it. If the heart needs more oxygen, it must either decrease the amount of work it is doing, utilize the oxygen more efficiently or increase the coronary flow rate. Usually the latter occurs. On the other hand, cerebral blood flow is slower and the brain removes only about $25 \%$ of oxygen delivered. Thus additional oxygen is immediately available if needed without an increase in blood flow.

Cerebral venous outflow. Generally, three sets of veins are described draining venous blood from the brain; the paired internal jugular veins, the multiple emissary veins, and the vertebral venous plexus. The role of the jugular and emissary veins is usually exaggerated and that of the vertebral plexus is ignored. There is evidence to indicate that the vertebral plexus may be the principle outflow tract when man is erect.

Flow of blood from the jugular vessels is controlled by intrathoracic pressure. Blood cannot escape from the jugular veins if intrathoracic pressure exceeds intravascular pressure. Yet even during the Valsalva manoeuvre, which can effectively stop outflow from the jugular vessels, blood continues to be pumped through the brain at the rate of $750 \mathrm{ml} / \mathrm{min}$. This blood cannot accumulate within the rigid skull. The route of escape is the vertebral plexus, a thin- walled valveless plexus extending from skull to pelvis, both within and adjacent to the intravertebral canal and with abundant connections with bony marrow spaces, subcutaneous vessels and deep veins in both chest and abdomen.

Cerebral venous blood will flow in the direction of the pressure gradient. Flow into the chest will be facilitated or impeded by changing intrathoracic pressure, and flow also will be affected by changes in body position. Cerebrospinal fluid is about 100 $\mathrm{mmH}_{2} \mathrm{O}$ pressure along the length of the spinal axis in the supine position. In the erect position, the cerebrospinal pressure increases in the lumbar area, simply from hydrodynamic effect and decreases within the skull. The cerebrospinal fluid has been measured at subatmospheric pressures beneath the vertex in the erect position. In addition to this negative pressure, the venous sinuses tend to remain patent due to their fibrous walls and connections to bone. Pressure within the cerebral and spinal veins may be assumed to parallel that of cerebrospinal fluid pressure at any given level. All of these factors together facilitate the flow of blood across the brain and, in fact, in the erect position a siphon effect may be one of our significant physiological compensations.

From the above it would appear that a slight head-up position is more physiological for cerebral circulation than is a head-down position. While in the latter position, the mean arterial perfusing pressure would be elevated, but so would the cerebrospinal fluid pressure and the cerebral venous pressure, both of which impede the flow of blood across the brain.

\section{References}

ECKENHOFF, J.E. (1970) The physiological significance of the vertebral venous plexus. Surgical Gynecology and $\mathrm{Ob}$ stetrics, 131, 72.

Epstein, H.M., Linde, H.W., Crampton, A.R., Ciric, L.S. \& ECKenhofF, J.E. (1970) The vertebral venous plexus as a major cerebral venous outflow tract. Anesthesiology, 32, 332.

Smith, A.L. \& Wollman, H. (1972) Cerebral blood flow and metabolism: effects of anesthetic drugs and techniques. Anesthesiology, 36, 378.

\section{Further reading}

Purves, M.J. (1972) The Physiology of the Cerebral Circulation. Cambridge University Press: London. 